\title{
Enhancing GABAergic Tone in the Rostral Nucleus of the Solitary Tract Reconfigures Sensorimotor Neural Activity
}

\author{
Joshua D. Sammons, ${ }^{1}$ Caroline E. Bass, ${ }^{2}$ Jonathan D. Victor, ${ }^{3}$ and Patricia M. Di Lorenzo ${ }^{1}$ \\ ${ }^{1}$ Department of Psychology, Binghamton University, Binghamton, New York 13902-6000, ${ }^{2}$ Department of Pharmacology and Toxicology, Jacobs \\ School of Medicine and Biomedical Sciences, Buffalo, New York 14203, and ${ }^{3}$ Feil Family Brain and Mind Research Institute, Weill Cornell Medical \\ College, New York, New York 10065
}

Recent work has shown that most cells in the rostral, gustatory portion of the nucleus tractus solitarius (rNTS) in awake, freely licking rats show lick-related firing. However, the relationship between taste-related and lick-related activity in rNTS remains unclear. Here, we tested whether GABA-derived inhibitory activity regulates the balance of lick- and taste-driven neuronal activity. Combinatorial viral tools were used to restrict the expression of channelrhodopsin 2-enhanced yellow fluorescent protein to $\mathrm{GAD}^{+}$GABAergic neurons. Viral infusions were bilateral in rNTS. A fiber-optic fiber attached to a bundle of drivable microwires was later implanted into the rNTS. After recovery, water-deprived rats were presented with taste stimuli in an experimental chamber. Trials were five consecutive taste licks $\left[\mathrm{NaCl}, \mathrm{KCl}, \mathrm{NH}_{4} \mathrm{Cl}\right.$, sucrose, monosodium glutamate/ inosine-5'-monophosphate, citric acid, quinine, or artificial saliva (AS)] separated by five AS rinse licks on a variable ratio 5 schedule. Each taste lick triggered a $1 \mathrm{~s}$ train of laser light $(25 \mathrm{~Hz} ; 473 \mathrm{~nm} ; 8-10 \mathrm{~mW})$ in a random half of the trials. In all, 113 cells were recorded in the rNTS, 50 cells responded to one or more taste stimuli without GABA enhancement. Selective changes in response magnitude (spike count) within cells shifted across-unit patterns but preserved interstimulus relationships. Cells where enhanced GABAergic tone increased lick coherence conveyed more information distinguishing basic taste qualities and different salts than other cells. In addition, GABA activation significantly amplified the amount of information that discriminated palatable versus unpalatable tastants. By dynamically regulating lick coherence and remodeling the acrossunit response patterns to taste, enhancing GABAergic tone in rNTS reconfigures the neural activity reflecting sensation and movement.

Key words: brainstem; GABA; gustatory; neural coding; nucleus of the solitary tract; taste

Significance Statement

The rostral nucleus tractus solitarius (rNTS) is the first structure in the central gustatory pathway. Electrophysiological recordings from the rNTS in awake, freely licking animals show that cells in this area have lick-related as well as taste-related activity, but the relationship between these characteristics is not well understood. Here, we showed evidence that GABA activation can dynamically regulate both of these properties in rNTS cells to enhance the information conveyed, especially about palatable versus unpalatable tastants. These data provide insights into the role of inhibitory activity in the rNTS.

\section{Introduction}

In mammals, information about taste is conveyed directly to the rostral nucleus tractus solitarius (rNTS). This structure directs taste information to higher-order structures, integrates information from

Received Feb. 18, 2020; revised Nov. 11, 2020; accepted Nov. 15, 2020.

Author contributions: P.M.D.L. designed research; J.D.S. performed research; J.D.S. and P.M.D.L. analyzed data; C.E.B. contributed unpublished reagents/analytic tools; P.M.D.L., J.D.S. and J.D.V. wrote the paper.

This work was supported by National Institute on Deafness and Other Communication Disorders Grant R01DC-006914 to P.M.D.L.

J.D. Sammons' present address: Department of Biochemistry and Molecular Genetics, University of Alabama at Birmingham, Birmingham, AL 35294-2170.

The authors declare no competing financial interests.

Correspondence should be addressed to Patricia M. Di Lorenzo at diloren@binghamton.edu.

https://doi.org/10.1523/JNEUROSCI.0388-20.2020

Copyright $\odot 2021$ the authors centrifugal sources, and, ultimately, influences movements aimed at ingestion. In rNTS of alert rats, only a minority of cells are taste responsive; most cells, including taste-responsive cells, track behavior (Denman et al., 2019). That is, when rats freely lick tastants of various qualities, coherence of firing patterns with the lick cycle is very common (Denman et al., 2019). The phase of the lick cycle associated with maximal firing varies widely from cell to cell, indicating that the lick-related responses are not simply motor signals or efference copy. Moreover, lick-related cells, by the arrangement of their spikes over time, also contribute information about taste quality along with canonically taste-responsive cells, albeit at a lower level (Roussin et al., 2012; Weiss et al., 2014; Denman et al., 2019). Thus, there is an intimate relationship between taste sensation and the movements associated with ingestion in the rNTS. 
The sensorimotor aspects of rNTS activity suggest that tasteresponsive cells collaborate with behavior-driven cells to encode taste; however, the extent to which taste responses can be altered experimentally or physiologically reveals a surprising amount of plasticity. For example, taste responsivity within a cell can be altered by taste adaptation (Di Lorenzo and Lemon, 2000), differences in taste context (Di Lorenzo et al., 2003), or the simple passage of time (Sammons et al., 2016), even to the point where taste responses that were not previously evident were uncovered. Further, suppression (Monroe and Di Lorenzo, 1995) or stimulation (Smith and $\mathrm{Li}, 2000$ ) of the gustatory cortex (GC), lateral hypothalamus (Matsuo et al., 1984; Murzi et al., 1986; Cho et al., 2002, 2003), and amygdala (AMG; Li et al., 2002; Cho et al., 2003), all of which provide centrifugal input to rNTS, can selectively alter responses to individual tastants in rNTS cells.

One potential mechanism that may underlie or contribute to these changes is the action of GABA, since several structures that send descending input to the rNTS either synapse on GABAergic interneurons (Smith and $\mathrm{Li}, 2000$ ) or provide GABAergic input directly to rNTS neurons (Saha et al., 2002).

The presence of GABA in the rNTS has been well documented (Lasiter and Kachele, 1988; Davis, 1993; Boxwell et al., 2013), but the functional consequences for taste coding are not fully understood. Leonard et al. (1999) argued that the localization of GABAergic terminals on dendrites in rNTS facilitates modulation of incoming gustatory signals. In physiological studies, Grabauskas and Bradley $(1998,1999)$ showed that tetanic stimulation of the solitary tract induces both short-term and long-term GABA-mediated potentiation of inhibitory synaptic activity, suggesting that this type of presynaptic plasticity may aid in stabilizing the response to afferent input (Grabauskas and Bradley, 1999). In addition to inhibition produced by afferent signals, taste-responsive cells in the rNTS are under tonic inhibitory influence (Smith and Li, 1998; Grabauskas and Bradley, 2003) presumably derived from GABAergic interneurons. The application of the GABA antagonist bicuculline can broaden the breadth of tuning of taste-responsive rNTS cells. Moreover, inhibitory interactions in rNTS may enhance and stabilize the temporal structure of taste-evoked spike trains (Rosen and Di Lorenzo, 2009). The caveat to what is known about GABAdriven inhibition in rNTS is that it is all derived from studies in anesthetized subjects; the function of inhibition in taste coding in awake subjects may be different.

Here, we tested the hypothesis that inhibition in the rNTS can modulate both the sensory-related and behavior-related activity in rNTS to alter taste coding. We used optogenetic tools to selectively enhance GABAergic activity in rNTS while rats freely licked taste stimuli. Results showed that GABA activation can selectively modify taste responses and can modulate lick coherence in a subset of cells. These changes reconfigured the relationship of sensory-related to motor-related activity in these cells, enhancing the information they conveyed about taste.

\section{Materials and Methods}

Subjects. Six male (weight, 250-450 g) and three female (weight, 200-350 g) Sprague Dawley rats obtained from Taconic Laboratories served as subjects. Of these, two males and two females served as nonviral control subjects. Food and water were provided ad libitum except during behavioral studies where rats were water deprived for $22-23 \mathrm{~h} / \mathrm{d}$. Rats were pair housed and maintained on a $12 \mathrm{~h}$ light/dark cycle with lights on at 9:00 P.M. All procedures were approved by the Institutional Animal Care and Use Committee of Binghamton University and conducted in accordance with the National Institutes of Health Guide for the Care and Use of Laboratory Animals.

Viral constructs and infusion. Rats were anesthetized with a ketamine/xylazine mixture (100:14 mg/kg, i.p.). Buprenorphine- $\mathrm{HCl}(0.05 \mathrm{mg}$, s.c.) was administered to enhance the effects of the anesthetic and atropine sulfate $(0.054 \mathrm{mg} / \mathrm{kg}$, s.c. $)$ to prevent excessive secretions. The scalp of the rat was shaved, and its head was secured in a stereotaxic instrument (David Kopf Instruments). The head was leveled with bregma and $\lambda$ in the same dorsal-ventral plane. The eyes of the rat were lubricated, and core temperature was maintained at $37^{\circ} \mathrm{C}$ with a heating pad attached to an anal thermistor probe. The scalp was then swabbed three times with Betadine alternated with $70 \%$ ethanol. An incision was made along the midline from bregma to the occipital ridge, and the skin and fascia were retracted with blunt dissection. A hole was drilled at $12 \mathrm{~mm}$ posterior and $\pm 1.75 \mathrm{~mm}$ lateral to bregma. A combination of viruses was infused $(0.5 \mu \mathrm{l}$ total; $0.5 \mu \mathrm{l} / \mathrm{min}$ ) bilaterally $6 \mathrm{~mm}$ below the surface of the brain. The combination consisted of $166 \mathrm{nl}$ of GAD1-Cre-AAV $2 / 10+333 \mathrm{nl}$ of Ef1 $\alpha$-DIO-ChR2-EYFP-AAV 2/10, which we have previously shown to restrict expression to $\mathrm{GAD1}^{+}$neurons (Xiao et al., 1998; Wakabayashi et al., 2019). All viruses were packaged using the triple transfection method to generate a pseudotyped virus, as detailed previously (Gompf et al., 2015). After each infusion, the needle was held in place for an additional 5 min to ensure complete expulsion of the virus. After retraction of the needle, the scalp was sutured and the rat allowed to regain consciousness. The animal was given a postoperative injection of buprenorphine- $\mathrm{HCl}$ $(0.05 \mathrm{mg}$, s.c.) and gentamicin $(0.05 \mathrm{mg}$, s.c.). Rats were allowed to recover for 2-4 weeks. Nonviral control rats $(n=4$; two male, two female) experienced the same surgical procedures as experimental rats but without viral infusion.

Optrode implantation surgery. Two to 4 weeks after viral infusion surgery, optrodes were implanted into the rNTS. Initially, rats were given buprenorphine- $\mathrm{HCl}(0.05 \mathrm{mg}$, s.c.) and atropine sulfate $(0.054 \mathrm{mg} / \mathrm{kg}$, s. c.). Animals were then anesthetized with $3 \%$ isoflurane in $\mathrm{O}_{2}$ at a flow rate of $0.9 \mathrm{~L} / \mathrm{min}$, and the scalp was shaved. Anesthesia was maintained with $1-3 \%$ isoflurane. The head of the rat was placed in a stereotaxic instrument (David Kopf Instruments) and swabbed with Betadine and $70 \%$ ethanol three times. The eyes were lubricated and the temperature of the rat was maintained at $37^{\circ} \mathrm{C}$ throughout the surgery. The skull was exposed from just anterior to bregma to $\sim 1.5 \mathrm{~cm}$ behind the occipital ridge. Five self-tapping screws were inserted into the skull. The head was angled with bregma $4 \mathrm{~mm}$ below $\lambda$, and a hole was drilled at 14.3-15.3 $\mathrm{mm}$ posterior and $1.7-1.8 \mathrm{~mm}$ lateral to bregma. The exposed dura was resected and an optrode consisting of 8 or 16 tungsten wires attached to a fiber-optic implant were lowered through the hole to $\sim 5-6 \mathrm{~mm}$ below the surface of the brain at a rate of $1 \mathrm{~mm} / 5 \mathrm{~min}$. The lower tip of the fiber-optic implant was positioned within $100 \mu \mathrm{m}$ of the tip of the microelectrode bundle. This arrangement ensured that the light stimulation impacted the neurons that were recorded (Yizhar et al., 2011). The 16channel electrode + fiber-optic bundles were drivable and placed $\sim 500 \mu \mathrm{m}$ above the rNTS. A ground wire was wrapped around one of the skull screws. The entire assembly was then embedded in dental acrylic. Rats were administered buprenorphine- $\mathrm{HCl}(0.05 \mathrm{mg}$, s.c. $)$ and gentamicin $(0.05 \mathrm{mg}$, s.c.) immediately following surgery and daily for 2 additional days. The rat was allowed to recover for $5 \mathrm{~d}$ or until it regained $90 \%$ presurgical body weight before testing began.

Apparatus. For an experimental session, rats were placed in an operant chamber (Med Associates) housed in an Multi-Density Fiberboard (MDF) outer box equipped with a house light and fan. One wall of the operant chamber had an opening that allowed access to a lick spout for the delivery of taste stimuli. The occurrence of a lick was detected when the rat broke an infrared beam as it accessed the lick spout. The stainless steel lick spout housed a collection of 16 stainless steel tubes for the delivery of 16 different taste stimuli. Reservoirs of taste stimuli were pressurized with air $(\sim 10 \mathrm{psi})$. Polyethylene $(\mathrm{PE})$ tubing connected the stimulus reservoirs to solenoids that, when activated by a computer signal, delivered $\sim 12 \mu \mathrm{l}$ of fluid to the lick spout through PE tubing attached to the stainless steel tubes in the lick spout.

Experimental paradigm. Rats were moderately water deprived (22$23 \mathrm{~h}$ ) and placed in the operant chamber where they had free access to 

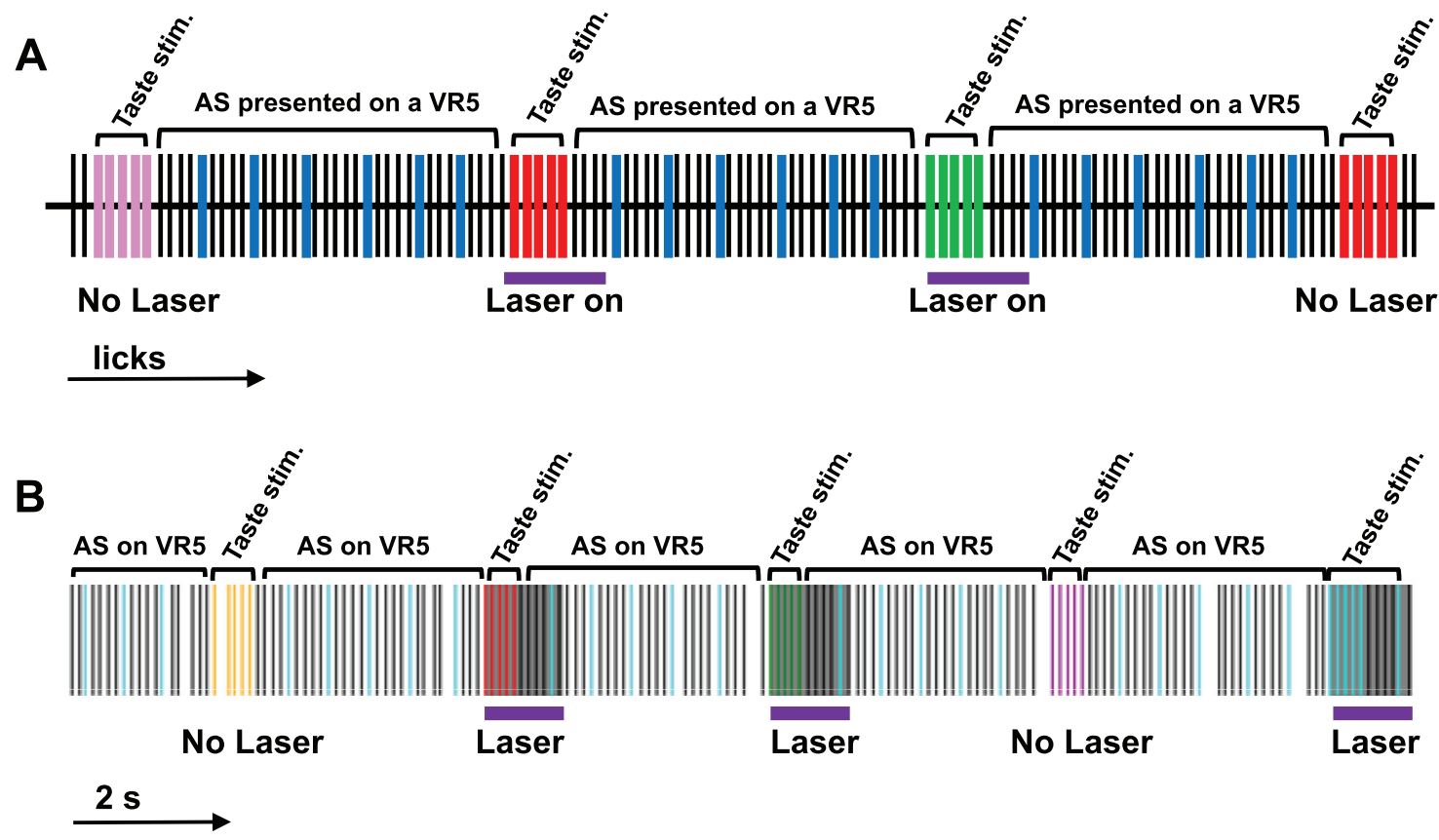

Figure 1. A, Schematic diagram of the experimental protocol. Each vertical line represents a lick. Colored lines represent reinforced licks; black lines represent dry licks. Taste stimuli were presented as five consecutive reinforced licks. A random half of the taste stimuli were accompanied by laser activation that lasted $1 \mathrm{~s}$ following each tastant lick. Between taste stimulus trials, six AS licks were presented on a VR5 schedule. Note that AS was used both as one of the taste stimuli and as the rinse for all of the tastants. $\boldsymbol{B}, \mathrm{A} 30 \mathrm{~s}$ sequence of licks from an actual test session showing taste stimulus presentations with and without laser. Note that in $\boldsymbol{A}$, the horizontal axis is schematic and shows the licks equally spaced; in $\boldsymbol{B}$, the horizontal axis is time, and the tickmarks indicate actual lick timing.

the lick spout for the entire experimental session $(30 \mathrm{~min})$. Taste stimuli consisted of $0.1 \mathrm{~m}$ sucrose, $0.1 \mathrm{M} \mathrm{NaCl}, 0.1 \mathrm{M}$ monosodium glutamate (MSG) plus $0.01 \mathrm{~m}$ inosine monophosphate, $0.1 \mathrm{M} \mathrm{KCl}, 0.1 \mathrm{M} \mathrm{NH}_{4} \mathrm{Cl}$, $0.01 \mathrm{~m}$ citric acid, $0.0001 \mathrm{M}$ quinine, and artificial saliva (AS; $0.015 \mathrm{M}$ $\mathrm{NaCl}, 0.022 \mathrm{M} \mathrm{KCl}, 0.003 \mathrm{M} \mathrm{CaCl}_{2} ; 0.0006 \mathrm{M} \mathrm{MgCl}_{2} ; \mathrm{pH} \sim 7.4$; Hirata et al., 2005; Breza et al., 2010). All tastants were reagent grade and dissolved in AS. (AS was presented as both a rinse and a taste stimulus.) The order of taste stimulus presentations was randomized. There were two types of licks: reinforced and dry. Each reinforced lick delivered $\sim 12 \mu \mathrm{l}$ of fluid. A taste trial consisted of five consecutive reinforced licks of a taste stimulus with no intervening dry licks. Between trials, five licks of an AS rinse were presented on a variable ratio 5 (VR5) schedule, with each reinforced AS lick occurring every four to six dry licks. During a randomly interspersed half of the taste stimulus trials, laser stimulation of GABAergic neurons $(473 \mathrm{~nm}$; $25 \mathrm{~Hz} ; 10-12 \mathrm{~mW}$ ) was triggered for $1 \mathrm{~s}$ after each reinforced stimulus lick. Figure $1 A$ shows a schematic of a typical sequence of taste stimulus trials (five consecutive reinforced licks) interspersed with rinse licks (presented on a VR5). Figure $1 B$ shows a sequence of licks over $30 \mathrm{~s}$ from an actual test session. The fiber-optic implant was static, but every 2-4 recording days, the microwires were extended ventrally $25-50 \mu \mathrm{m}$. Experimental sessions were $30 \mathrm{~min}$ in length and continued daily, except for weekends, for 2-4 weeks.

Electrophysiological recording and light stimulation. During the experimental session, the electrode bundle of the rat was connected to an Omniplex D. Neural Data Acquisition System (Plexon). Timing for electrophysiological activity and stimulus events were recorded using PlexControl software (Plexon). The fiber-optic implant was attached to a $473 \mathrm{~nm}$ laser source (Shanghai Laser and Optics Century Co, Ltd.) through a fiber-optic patch cable $(1 \mathrm{~m}$ length, $200 \mu \mathrm{m}$ core, 0.22 numerical aperture; THORLABS). Optic stimulation was triggered in a random half of tastant trials, as mentioned in the Experimental paradigm section.

Neuronal signals were isolated in Offline Sorter (Plexon) or through a semisupervised spike sorting Python program adapted from Mukherjee et al. (2017; https://github.com/dmarshall-bing/AutoSort). Less than $0.5 \%$ of waveforms contained an interspike interval $<1 \mathrm{~ms}$.
Analyses of taste responses. Spontaneous firing rate was calculated as follows: first, periods where the rat was not licking for at least $10 \mathrm{~s}$ were identified. Next, the first $3 \mathrm{~s}$ and the last $1 \mathrm{~s}$ of activity during that period were discarded to ensure that the remnants of a lick bout or preparation for a lick bout were not included as spontaneous activity. Finally, firing rates during these periods without licking were pooled and divided into $1 \mathrm{~s}$ intervals, and the overall firing rate calculated in spikes per second (sps).

As in previous work (Roussin et al., 2012; Weiss et al., 2014; Escanilla et al., 2015), responses to taste stimuli were detected over the following two time scales: (1) responses that extended across more than one lick, called "five-lick" responses; and (2) responses that occurred briefly after each lick, called "lick-by-lick" responses.

Five-lick responses were quantified by a significant increase or decrease in firing rate over five consecutive taste licks (without intervening unreinforced licks), compared with baseline firing rate for at least $300 \mathrm{~ms}$. Baseline firing rate was calculated in $100 \mathrm{~ms}$ time bins over the $1 \mathrm{~s}$ preceding the first taste stimulus lick in a trial. To determine whether a significant response was present, the firing rate in $100 \mathrm{~ms}$ time bins, beginning with the first taste stimulus lick, was compared with the $95 \%$ confidence limits of the baseline firing rate. The $100 \mathrm{~ms}$ window was moved in $20 \mathrm{~ms}$ increments until there were at least three consecutive, nonoverlapping $100 \mathrm{~ms}$ bins where there was a significant difference between baseline and response firing rates. The leading and trailing edge of the significant bins were used to determine when a taste response started (latency) and ended (duration), respectively. A maximum of two bins within a response was allowed to be nonsignificant. The response magnitude (firing rate during a response minus the baseline firing rate), latency, duration, and baseline activity were calculated for each taste response. Neurons with a response spike rate of $<2$ sps were not included.

Lick-by-lick responses were detected using a $\chi^{2}$ test comparing responses from the average spike rate of the last nonreinforced lick (i.e., a dry lick) before every tastant trial to the average spike rate of every lick from each tastant. Response windows were limited to $150 \mathrm{~ms}$ after each lick and divided into $1015 \mathrm{~ms}$ bins. We chose the interval of $150 \mathrm{~ms}$ to measure lick-by-lick responses because this was the median interlick 
interval overall. We chose $15 \mathrm{~ms}$ bins because not every lick-by-lick response spanned the full $150 \mathrm{~ms}$. Had we just used the entire $150 \mathrm{~ms}$ interlick interval to measure the responses, we might have missed some very brief but significant responses that occurred following each lick of a taste stimulus. Using the $\chi^{2}$ test, the actual response value from each bin of each tastant versus dry lick was compared with the corresponding expected response bin. A Bonferroni correction was made for multiple ( $n=16$ tastants; i.e., 8 tastants with and 8 tastants without GABA activation) comparisons. Neurons with a response firing rate $<4$ sps during lick bouts were excluded.

To test for the effects of GABA stimulation on taste responses, we performed a $\chi^{2}$ analysis of the peristimulus time histograms (PSTHs; $100 \mathrm{~ms}$ bins from $t=0$ to $t=4 \mathrm{~s}$ following the first taste stimulus lick) and compared GABA versus non-GABA stimulation to obtain a $p$-value. We then corrected for multiple comparisons using the false discovery rate method (Benjamini and Hochberg, 1995).

Analysis of breadth of tuning. In addition to noting the number of tastants to which a cell responds, the breadth of tuning was assessed by calculating two standard measures of tuning breadth that are more graded: taste entropy and taste sharpness. Each measure reflects a different aspect of tastant specificity. Both analyses were performed using the five prototypical tastants (sucrose, $\mathrm{NaCl}, \mathrm{MSG}$, citric acid, and quinine). Taste entropy (Smith and Travers, 1979) is a measure of uncertainty based on the similarity of response magnitudes between tastants. This measure is calculated as follows:

$$
H=-k \sum_{i=1}^{n} P_{i} \log P_{i}
$$

where $n$ is the number of tastants (five), $k=1.4307$ for five tastants, and $P_{i}$ is the ratio of tastant $i$ response magnitude to the sum of all tastant response magnitudes. The value ranges from 0 , signifying that the neuron responds to a single tastant, to 1 , indicating that the neuron responds to all five tastants equally. Taste sharpness (Rainer et al., 1998) is a measure of how similar taste magnitudes are to the best stimulus and is calculated as follows:

$$
\text { Sharpness }=\frac{\left(n-\sum_{i=1}^{n} T_{i} / T_{\text {best }}\right)}{n-1},
$$

where $n$ is the number of tastants, $T_{i}$ is response magnitude for tastant $i$, and $T_{\text {best }}$ is the response magnitude of the best stimulus. Similar to the entropy measure, a value of 0 indicates a response to a single tastant, and a value of 1 indicates equal responses to all five tastants.

Temporal coding analysis. Analysis of information about taste quality conveyed by individual neurons was performed using metric space analyses (MSAs; Victor and Purpura, 1996, 1997). This method has been described in detail previously (Roussin et al., 2012; Weiss et al., 2014; Escanilla et al., 2015; Sammons et al., 2016) and is only summarized here. The basic approach of MSA is to measure the "cost" of converting one spike train (e.g., a response to a tastant) into another as a measure of similarity/dissimilarity. Cost is accrued by insertion or deletion of spikes or movement of spikes in time. The insertion or deletion of a spike costs one arbitrary unit. Movement of a spike in time costs $q t$ units, where $q$ is a parameter of temporal precision ( $1 / q$ has units of seconds), and $t$ is the amount of time that the spike is shifted. Thus, at $q=0$, the cost of moving a spike is 0 , so spike timing is ignored when comparing spike trains; as $q$ increases, spike timing is taken into account with progressively greater precision. At each value of $q$, the mutual information $H$ between tastants and neural responses is estimated by comparing the similarity of pairs of responses to the same stimulus with the similarity of responses to different stimuli. To mitigate biases because of sample size, the Treves-Panzeri-Miller-Carlton debiaser was applied to all estimates of $H$ (for review, see Panzeri et al., 2007). This computation of information

\begin{tabular}{|c|c|c|c|c|c|}
\hline Stimulus & Trials & ILI (s) & No. of pauses & Pause length (s) & 5 licks (s) \\
\hline Sucrose & 200 & 0.155 & 2 & 1.891 & 0.644 \\
\hline Sucrose + GABA & 203 & 0.155 & 2 & 2.160 & 0.642 \\
\hline $\mathrm{NaCl}$ & 194 & 0.150 & 2 & 1.278 & 0.625 \\
\hline $\mathrm{NaCl}+\mathrm{GABA}$ & 204 & 0.151 & 1 & 1.091 & 0.627 \\
\hline Citric acid & 208 & 0.155 & 14 & 1.826 & 0.653 \\
\hline Citric acid + GABA & 202 & 0.156 & 14 & 1.923 & 0.655 \\
\hline Quinine & 211 & 0.158 & 30 & 9.005 & 0.668 \\
\hline Quinine $+G A B A$ & 205 & 0.157 & 27 & 1.622 & 0.657 \\
\hline MSG & 204 & 0.147 & 3 & 1.730 & 0.628 \\
\hline$M S G+G A B A$ & 201 & 0.148 & 0 & NA & 0.622 \\
\hline $\mathrm{KCl}$ & 206 & 0.153 & 13 & 1.483 & 0.627 \\
\hline $\mathrm{KCl}+\mathrm{GABA}$ & 200 & 0.155 & 9 & 1.281 & 0.646 \\
\hline $\mathrm{NH}_{4} \mathrm{Cl}$ & 199 & 0.147 & 11 & 1.227 & 0.616 \\
\hline $\mathrm{NH}_{4} \mathrm{Cl}+\mathrm{GABA}$ & 202 & 0.148 & 8 & 2.156 & 0.623 \\
\hline AS & 207 & 0.161 & 19 & 1.663 & 0.667 \\
\hline$A S+G A B A$ & 201 & 0.161 & 9 & 3.729 & 0.680 \\
\hline
\end{tabular}

Table 1. Analyses of lick behavior

Trials, Total number of trials for each stimulus across animals; ILI, median interlick interval; No. of pauses, the median number of pauses within a 5 -lick taste stimulus trial; Pause length, the median duration (in seconds) of pauses that occurred within a five-lick taste stimulus trial; Five licks, the median time (in seconds) to complete all five licks of a stimulus trial.

conveyed about taste quality is conducted across a range of values of $q$, and the maximum is denoted as $H_{\max }$.

Two auxiliary analyses using synthetic data were also conducted. First, to account for residual bias in the estimation of information, spike trains for 40 pairs of randomly labeled responses were compared using MSA; this yields $H_{\text {shuffled. }}$ Second, to determine whether temporal information was because of spike timing per se, versus differences in the rate envelope, spikes within each taste-evoked spike trains were randomly assigned to alternative responses to the same tastant, while preserving the rate envelope; calculation of information from these synthetic datasets this yields $H_{\text {exchange }}$. Information about taste quality conveyed by spike timing was considered significant only if $H_{\max }>H_{\text {shuffled }}+2$ SDs and $H_{\max }>$ $H_{\text {exchange. }}$. If $H_{\max }>H_{\text {shuffled }}+2 \mathrm{SDs}$, but not $H_{\text {exchange, }}$ information was considered significant, but information conveyed by spike timing was not considered significant. Information from neurons where $H_{\text {max }} \leq H_{\text {shuffled }}+2$ SDs was set to 0 .

To characterize the information conveyed by the population of cells, we calculated the average amount of information conveyed by the entire sample of units at $200,500,1000,1500$, and $2000 \mathrm{~ms}$ of the cumulative response. Information conveyed by the lick pattern was determined in the same way as for spike trains, and was compared with that conveyed by spike trains. Only neurons from sessions that contained at least six taste trials with and six taste trials without laser stimulation were included in the temporal coding analysis.

Statistical analyses of lick coherence. For the firing pattern of each neuron, its coherence with the occurrence of licks was calculated using the NeuroExplorer 5.201 Coherence Analysis function (NexTechnologies). Single-taper Hann windowing was used to calculate the values of 256 frequency bins between 0 and $50 \mathrm{~Hz}$ frequency with a $50 \%$ overlap between windows. The analysis calculates confidence as described in the study by Kattla and Lowery (2010). Neurons with a coherence value $>99 \%$ confidence between 4 and $9 \mathrm{~Hz}$ were considered lick coherent. In lick-coherent neurons, differences in lick coherence were obtained around tastant licks with laser stimulation versus tastant licks without laser stimulation. The reported difference in coherence value was calculated as the maximum difference in coherence between 4 and $9 \mathrm{~Hz}$. An $F$ test was used to determine whether the change in coherence observed between baseline conditions without GABA activation and during GABA activation was actually because of GABAergic activation or random chance.

Spearman's rank correlation coefficient $(\rho)$ was calculated to determine correlations between lick coherence and measures of taste specificity. The two-tailed $p$ value for each value was obtained for each correlation and a Bonferroni correction was made for multiple $(n=6)$ comparisons. The six different comparisons were 

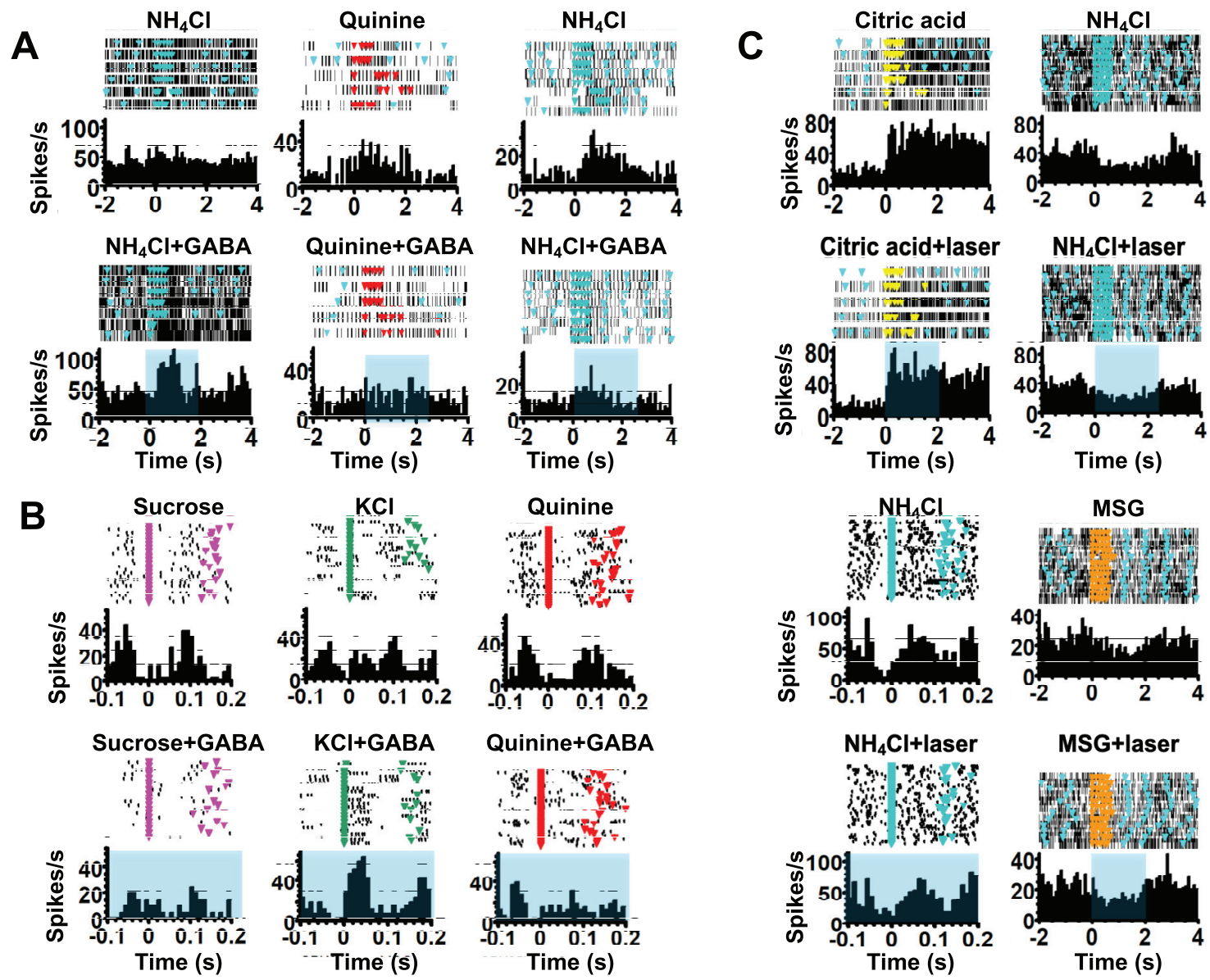

Figure 2. Examples of the effects of GABA activation on taste stimuli in six different cells. $A, B$, Top row, Responses without GABA stimulation. Bottom row, Responses with GABA enhancement. The top of each panel shows raster plots of each trial, with black lines/dots signifying the occurrence of a spike. Colored triangles indicate reinforced licks. Light blue triangles show AS licks. The bottom of each panel shows a PSTH of the response, in spikes per second. Shaded area indicates the presence of the laser. For lick-by-lick responses, the laser is presented for the entire lick-by-lick response. $\boldsymbol{A}$, Examples of five-lick responses with and without GABA stimulation in three different cells. $\boldsymbol{B}$, Examples of lick-by-lick responses from three different cells with and without GABA stimulation. C, Examples of the effects of laser application in the rNTS in animals that were not infused with virus.

lick coherence versus taste tuning, taste entropy, and taste sharpness, each with and without GABA stimulation.

Histology/immunolabeling. Rats were killed with sodium-pentobarbital ( $390 \mathrm{mg} / \mathrm{kg}$, i.p.). Just before expiration, $10 \mathrm{~s}$ of $1 \mathrm{~mA} \mathrm{DC}$ current was passed through the microwire with the last taste response. The rat was then transcardially perfused with isosaline followed by $4 \%$ paraformaldehyde (PFA) in PBS $(1 \times$ PBS). The brain was extracted and placed in $4 \%$ PFA overnight. The next day, brains were washed three times with PBS and stored in $20 \%$ sucrose in $1 \times$ PBS. Brains were then sectioned into $35 \mu \mathrm{m}$ coronal slices. Every other section was individually placed into wells of a 96-well dish containing a cryoprotectant (30\% ethylene glycol, $30 \%$ glycerol, 11.4 $\mathrm{mm} \mathrm{NaH}_{2} \mathrm{PO}_{4}-\mathrm{H}_{2} \mathrm{O}$, and $\left.38.4 \mathrm{~mm} \mathrm{Na} \mathrm{NPO}_{4}\right)$. The other half of the sections was placed directly onto Superfrost Plus slides and stained with cresyl violet for lesion site identification. The center of each lesion was taken as the final site of recording.

Sections placed into the cryoprotectant were removed and washed three times with $1 \times$ PBS. They were placed in blocking agent [ $10 \%$ bovine serum albumin (BSA), $0.1 \%$ Triton $\mathrm{X}, 1 \times \mathrm{PBS}$ ] and gently rocked for $1 \mathrm{~h}$ at room temperature (RT). Sections were then placed in primary $[10 \%$ BSA; $1: 1000$ rabbit anti-GFP (catalog \#AB290, Abcam); 1:500 mouse anti-NeuN (catalog \#MAB377, Millipore); $1 \times$ PBS] for an additional $2 \mathrm{~h}$ at RT or overnight at $4^{\circ} \mathrm{C}$. Sections were washed three times with $1 \times$ PBS and placed in secondary (1:500; AF488-conjugated goat anti-rabbit; catalog \#AB150077, Abcam); 1:500 Cy3 conjugated donkey anti-mouse (catalog \#715-165-151, Jackson ImmunoResearch); 1:10,000 DAPI stain (catalog \#5.08,741.0001, Millipore), $1 \times$ PBS] for $1 \mathrm{~h}$ at RT.

\section{Results}

\section{General response characteristics}

We recorded 113 isolated neurons from the rNTS of five freely licking rats (four male and one female) with optrode implants. Without GABA stimulation, a total of 50 (of $113 ; 44 \%$ ) neurons responded to at least one of the eight taste stimuli tested. With GABA stimulation, 43 (of $113 ; 38 \%$ ) neurons responded to at least one of the eight taste stimuli. Four neurons were unresponsive without GABA stimulation but showed taste responses with GABA stimulation resulting in a total of 54 neurons that responded to at least one tastant either with or without GABA activation. Of the 54 recorded taste neurons, 52 (96\%) responded to the five prototypical tastants (sucrose, $\mathrm{NaCl}, \mathrm{MSG}$, citric acid, or quinine), while two only responded with inhibitory lick-bylick responses to artificial saliva with GABA stimulation.

In all, there was no effect of laser stimulation in these nonviral control animals. There were 26 neurons, 15 of these taste responsive, that were recorded in four nonviral control animals (two male, two female). In addition, there were 10 recorded channels in these animals that contained evidence of several cellular waveforms above the noise level that could not be isolated as single units but collectively showed taste responses but no effect of laser.

The average spontaneous firing rate for the population was $18.4 \pm 2.8 \mathrm{sps}$ (median $=5.6 \mathrm{sps}$ ). The spontaneous firing rate for 
taste responsive neurons (mean $=16.5 \pm 4.3$ sps; median $=5.2 \mathrm{sps}$ ) was not significantly different from the spontaneous firing rate for nontaste neurons (mean $=20.1 \pm 3.7 \mathrm{sps}$; median $=7.0 \mathrm{sps}$ ). When animals began licking, the overall firing rate decreased for 19 $(17 \%)$ of the neurons and increased for 39 (35\%) of the neurons, regardless of whether the licks were reinforced or not.

Analyses of licking behavior

To assess the potential effects of GABAergic stimulation on licking behavior, we examined the microstructure of licking for each taste stimulus with and without laser stimulation. Table 1 shows the results of those analyses. Median interlick intervals, measured during the five-lick taste trial did not significantly differ when the taste-reinforced licks were presented with or without GABA stimulation (Wilcoxon signedrank test, $p=0.188)$. Moreover, there was no significant difference in the median number of pauses during the five-lick taste trial (Wilcoxon signed-rank test, $p=0.336)$ and no significant difference in the pause length (Wilcoxon signed-rank test, $p=0.453$ ). Finally, there was no significant difference in the total time to complete the five-lick taste trials across tastants (Wilcoxon signed-rank test, $p=$ 0.945). In all, these results suggest that GABA activation during taste acquisition did not alter lick patterns per se.

\section{GABAergic stimulation changed taste} profiles of individual neurons

Figure 2, illustrating taste responses from six different cells with and without GABA activation, shows that GABA activation selectively modified taste response magnitudes in 22 (of 54; 41\%) rNTS cells. GABA stimulation sometimes enhanced responses (Fig. 2A,B), even when there were no responses without GABA activation. Conversely, the activation of GABA attenuated or eliminated responses to taste stimuli at other times (Fig. $2 A, B$ ). There were 10 occasions where GABA activation enhanced some stimuli and attenuated others in the same cell. Figure $2 C$ shows the responses of four different cells illustrating the lack of an effect of the laser in nonviral control animals.

Taste response magnitudes with and without GABA activation for all taste-responsive cells are shown in Figure 3; both enhancement and attenuation of taste responses by GABA were evident across cells. Stimulation of GABA release in the rNTS changed taste-response magnitudes for both five-lick (Fig. $3 A$ ) and lick-by-lick responses (Fig. $3 B$ ). Neurons in this figure were organized according to their best stimulus (i.e., the stimulus that evoked the largest response without GABA stimulation; gray bars). Responses to tastants with GABA stimulation are overlaid as diamonds. Table 2 summarizes the stimulus-by-stimulus effects of GABA activation on rNTS cells.
Table 2. Effect of GABA stimulation on taste response magnitudes in rNTS

\begin{tabular}{lrrrrrrrr}
\hline & Sucrose & $\mathrm{NaCl}$ & MSG & Citric acid & Quinine & $\mathrm{KCl}$ & $\mathrm{NH}_{4} \mathrm{Cl}$ & $\mathrm{AS}$ \\
\hline $\begin{array}{l}5 \text {-Lick response* } \\
\quad \text { Increased }\end{array}$ & 0 & 1 & 1 & 1 & 3 & 1 & 1 & 0 \\
$\quad$ Decreased & 2 & 1 & 0 & 1 & 0 & 1 & 1 & 2 \\
$\quad$ No change & 10 & 15 & 12 & 12 & 9 & 8 & 14 & 4 \\
Lick-by-lick** & & & & & & & & \\
$\quad$ Increased & 3 & 2 & 2 & 0 & 2 & 0 & 2 & 4 \\
$\quad$ Decreased & 0 & 3 & 2 & 1 & 3 & 0 & 1 & 2 \\
$\quad$ No Change & 20 & 20 & 21 & 19 & 15 & 11 & 22 & 20 \\
All responses*** & & & & & & & & \\
$\quad$ Increased & 2 & 2 & 3 & 1 & 5 & 1 & 4 & 3 \\
$\quad$ Decreased & 2 & 4 & 2 & 1 & 3 & 1 & 1 & 4 \\
$\quad$ No Change & 26 & 29 & 28 & 29 & 22 & 16 & 28 & 23 \\
\hline
\end{tabular}

$* 10$ of $27(37 \%)$ cells affected; $* * 16$ of 41 (39\%) cells affected; $* * * 22$ of 54 (41\%) cells affected. 
Without +GABA

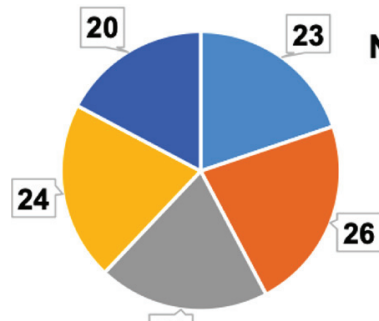

23

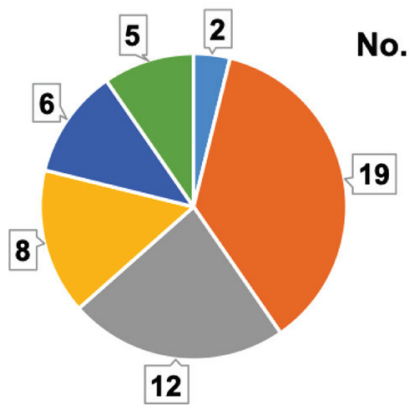

With +GABA
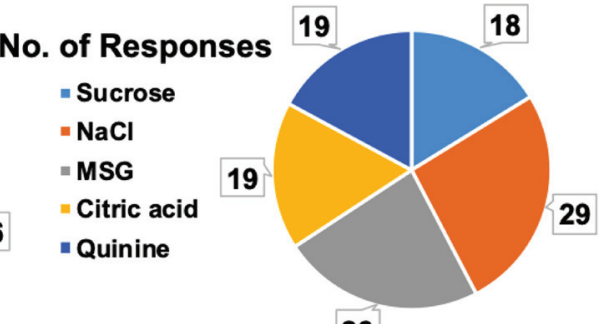

26

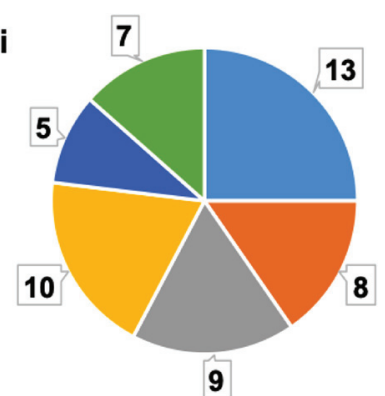

Figure 4. Pie graphs showing the distribution of the number of responses to each of the five basic tastants (top) and the number of tastants to which a neuron responds (bottom). Graphs on the left are distributions without GAB activation; graphs on the right show the distributions with GABA activation. Although each tastant evoked about the same number of responses across the sample, neurons were significantly more narrowly tuned with GABA stimulation. See text for details.

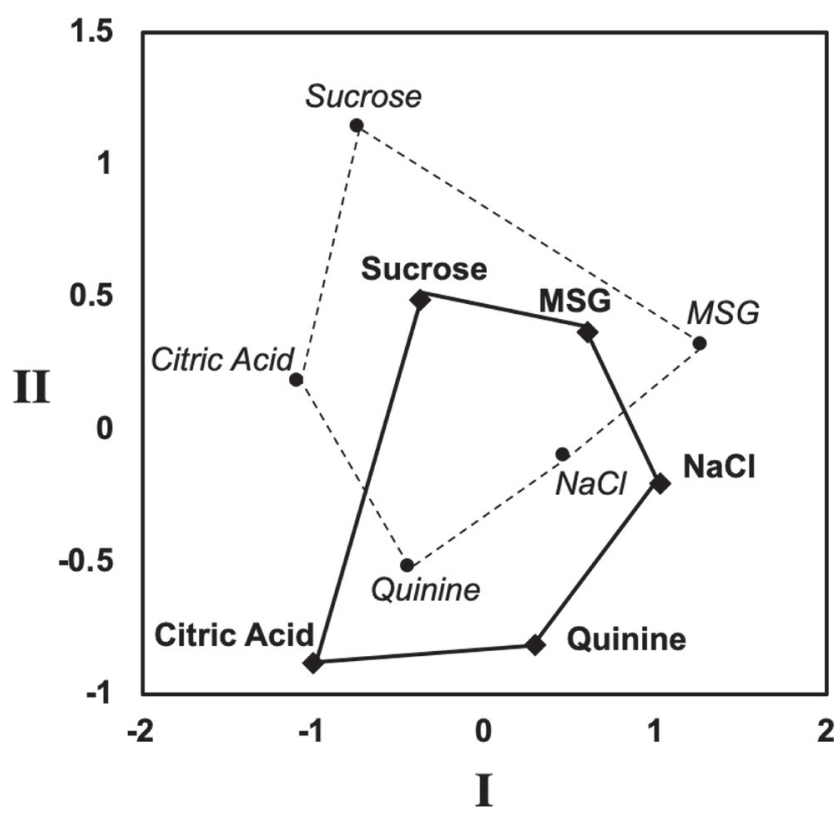

Figure 5. Multidimensional scaling of taste response magnitudes. Pearson's correlations were used as a measure of similarity for across-unit patterns evoked by tastants. A dashed line connects the patterns evoked by taste stimuli without GABA stimulation; a solid line connects the patterns evoked by taste stimuli with GABA stimulation. Gutman stress values were as follows: one dimension, 0.281 ; two dimensions, 0.108 ; three dimensions, 0.062 ; four dimensions, 0.026; five dimensions, 0.017 . GABA activation shifted the location of all tasteevoked across-unit patterns, but the overall organization was unchanged.

Based on previous studies that have suggested a role for GABA in modulating the breadth of tuning in brainstem tasteresponsive cells (Smith et al., 1998; Smith and Li, 1998), we analyzed the effects of GABA enhancement in rNTS cells using three

complementary approaches. First, we examined the number of tastants, each representing a basic taste quality, to which rNTS responded before and after GABA activation. Results showed that GABA enhancement reduced the number of tastants to which a neuron responded, consistent with previous reports in the literature (Smith and Li, 1998). This is illustrated in Figure 4. Although the total number of responses to any given tastant was not altered by GABA stimulation $\left(\chi^{2}=1.46, \mathrm{df}=4\right.$, $p=0.835)$, the number of tastants to which individual neurons responded decreased significantly $\left(\chi^{2}=13.62, \mathrm{df}=5, p=0.018\right)$. This was largely because of an increase in the number of cells that were rendered unresponsive or only responded to a single stimulus with GABA activation. Our second approach was the uncertainty measure (Smith and Travers, 1979), which did not show a significant difference with or without GABA activation. Specifically, the average taste uncertainty was $0.37 \pm 0.05$ without GABA stimulation and $0.48 \pm 0.05$ (Student's $t$ test, $p=0.535$ ) with GABA stimulation. Finally, our third approach was the taste sharpness measure, which also did not differ significantly following GABA activation. The average taste sharpness was $0.81 \pm 0.03$ without GABA stimulation and $0.76 \pm 0.03$ with GABA stimulation (Student's $t$ test, $p=0.698$ ). In sum, results show that GABA activation reduced the number of tastants to which a subset of units responded, resulting in changes in the breadth of tuning; however, across the population, there was no net effect of GABA activation on taste tuning (measured by the uncertainty and sharpness measures). Essentially, responses to various taste stimuli were redistributed across the population.

\section{Effect of GABA on across-unit patterns}

To determine whether GABA activation altered the pattern of responses to the tastants at the population level, we applied a multidimensional scaling analysis using Pearson correlations as measures of similarity. A hypothetical "taste space" placed the across-unit patterns for each taste stimulus close together or far apart depending on their similarity/dissimilarity. Across-unit response patterns both before and during GABA activation were analyzed and graphed together. Figure 5 shows the results of the combined analysis. Without GABA activation, response patterns to each of the five basic were well separated in taste space, suggesting that each tastant evoked easily discriminable patterns of response. With GABA activation, the configuration of acrossunit patterns was similar but shifted in space, indicating that the basic interrelationships among tastant-evoked response patterns was intact, but the identities of the units that contributed most to the pattern were different.

\section{GABA alters lick coherence, especially in taste neurons}

As coherence with licking is a prominent aspect of firing patterns in the NTS and points to integration of sensory and motor activity, we next asked whether this coherence is modulated by GABA. In our sample, the majority of rNTS neurons (97 of 113; $86 \%)$ were coherent with licking. Coherence values associated with all licking within a session will be termed overall lick coherence. In general, overall lick coherence values for taste neurons 
were significantly higher than those of nontaste neurons $(p<0.001$; taste neurons: mean $=2.2 * 10^{-1}$; median $=1.7 *$ $10^{-1} ; n=54$; nontaste neurons: mean $=6.3$ $* 10^{-2} ;$ median $=2.4 * 10^{-2} ; n=59$ ).

To determine whether and how GABA activation affected lick coherence, we restricted coherence analysis to licks that resulted in taste stimulus delivery, since this is when GABA release was triggered. Coherence values associated with licking only during tastant delivery will be termed tastant-restricted lick coherence. Not surprisingly, tastant-restricted lick coherence values without GABAergic stimulation were also higher in taste neurons than in nontaste neurons $(p<0.001$; taste neurons: mean $=3.3 * 10^{-2}$; median $=2.3 *$ $10^{-2}$; nontaste neurons: mean $=1.0 *$ $10^{-2}$; median $=5.2 * 10^{-3}$ ). The distribution of lick coherence values for both the global lick coherence and tastant-restricted lick coherence can be seen on the abscissas of Figure $6, A$ and $B$, respectively. Figure $6 B$ additionally tracks the change in tastant-restricted lick coherence on GABAergic stimulation over the ordinate and shows that tasteresponsive neurons are affected to a greater degree than nontaste neurons.

\section{GABA activation increased gustatory information in rNTS neurons}

To analyze the effect of enhancing GABAergic tone on temporal coding of taste stimuli, we applied MSA to datasets with at least six repetitions of each tastant (with and without GABA activation). Sixty neurons (38 taste responsive; 22 non-taste responsive) were included in these analyses; as previously noted (Denman et al., 2019), neurons that are not considered "taste responsive" by classical criteria nevertheless may carry information about taste when analyzed by MSA. That is, some aspect of their firing patterns (e.g., lick relatedness) may convey information about taste quality identity, even if the overall firing rate does not have a detectable dependence on tastant.

Among the 38 taste-responsive neurons, GABA stimulation reduced taste-related information to 0 in 10 and generated significant taste-related information in 14 (Fig. $7 A$ ). GABA activation eliminated taste-related information from 9 of the 22 non-tasteresponsive neurons and generated information from five nontaste-responsive neurons (Fig. $7 B$ ). (Note that, as detailed in Materials and Methods, when the information conveyed about taste quality in a given neuron was not significantly different from that in the randomly shuffled control, we set "information" at 0 .)

Taste-related information conveyed by spike timing was also analyzed at various response intervals ranging between $200 \mathrm{~ms}$ and $2 \mathrm{~s}$. Figure 8 shows the results of those analyses. At $2 \mathrm{~s}$, GABA activation increased taste-related information on average by 0.11 bits ( $48 \%$ increase) when all cells with five-lick taste responses were considered. To determine the relationship of these changes in information to changes in lick coherence, we divided all the neurons $(n=113)$ into quartiles based on the change in GABA-evoked changes in taste-restricted lick coherence. For the five-lick taste neurons $(n=27), 8$ fell into the "decrease coherence" group, 11 fell into the "increase coherence" group, and 8 fell into the "no change in coherence" group (middle two quartiles). For the lick-by-lick taste neurons $(n=41), 17$ decreased coherence with GABA stimulation, 13 increased coherence, and 11 had no change in coherence on GABA stimulation.

Cells in the uppermost quartile in which GABAergic stimulation increased lick coherence $(n=24)$ had a consistent increase in taste information with a maximum increase of 0.16 bits $(68 \%$ increase) at $2 \mathrm{~s}$ with GABA activation. Taste-related information was not affected by GABA stimulation in neurons in the bottom three quartiles. Thus, the increase in taste-related information associated with GABA activation was primarily carried by the neurons whose lick-related activity was most increased by GABA. Further, information on the lick patterns of different taste qualities of the rats was overall slightly decreased by GABA activation, suggesting that changes in the lick pattern per se cannot account for the increased taste quality information.

\section{Information about salty tastants is increased after GABAergic stimulation}

In addition to the five prototypical tastants, we also tested $\mathrm{KCl}$ and $\mathrm{NH}_{4} \mathrm{Cl}$ to determine whether GABA stimulation would increase the distinction between tastants of the same taste quality. Over the entire population of neurons, GABAergic stimulation had no effect on information relayed on the salty tastants (Fig. 9). However, when broken into the effect of GABAergic stimulation on lick coherence, similar to information about taste qualities, information relayed about the salty tastants was increased when lick coherence was also increased (Fig. 9), with a maximum increase of 0.13 bits $(51 \%)$ at $1.5 \mathrm{~s}$. Similar to taste quality analysis, GABAergic stimulation had no effect on neurons with decreased coherence or no change in coherence, and it had no effect on information from the lick pattern.

Effect of GABA activation on information about palatability Much of the information increase obtained by GABA stimulation occurs a second or two after tastant delivery is initiated. This time epoch is thought to signal taste palatability, at least in the gustatory cortex (Katz et al., 2001). As such, we sought to determine whether GABAergic stimulation would increase information about the palatability of tastants. We collapsed responses to sucrose and $\mathrm{NaCl}$ as the palatable tastants and collapsed responses to citric acid and quinine as the nonpalatable tastants 

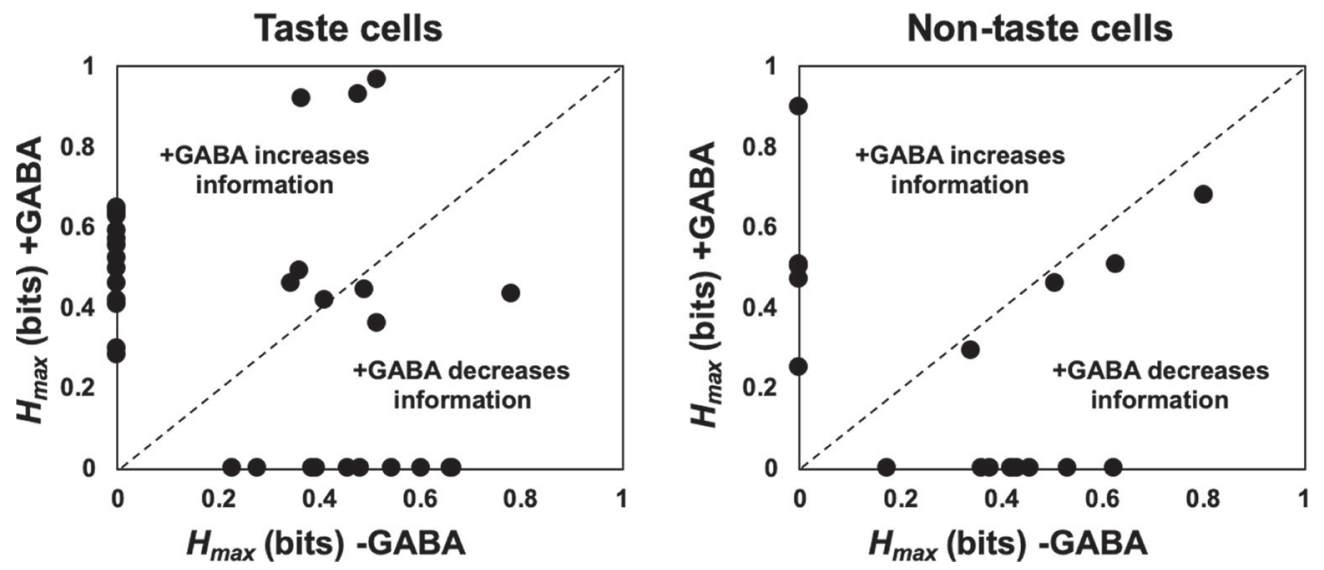

Figure 7. Information (in bits) conveyed about taste quality in taste and nontaste neurons in rNTS. For each cell, $H_{\max }$ was plotted without versus with laser-stimulated GABA release. Taste cells (left) or non-taste cells (right). Only neurons with at least six trials for each tastant were used. Information was conveyed by the temporal aspects of taste responses in 33 of 54 (61\%) taste cells and 18 of $59(31 \%)$ non-taste cells. GABA stimulation either enhanced or attenuated information conveyed about taste quality in $73 \%$ taste cells (24 of 33 ) and $78 \%$ of nontaste cells (4 of 18).

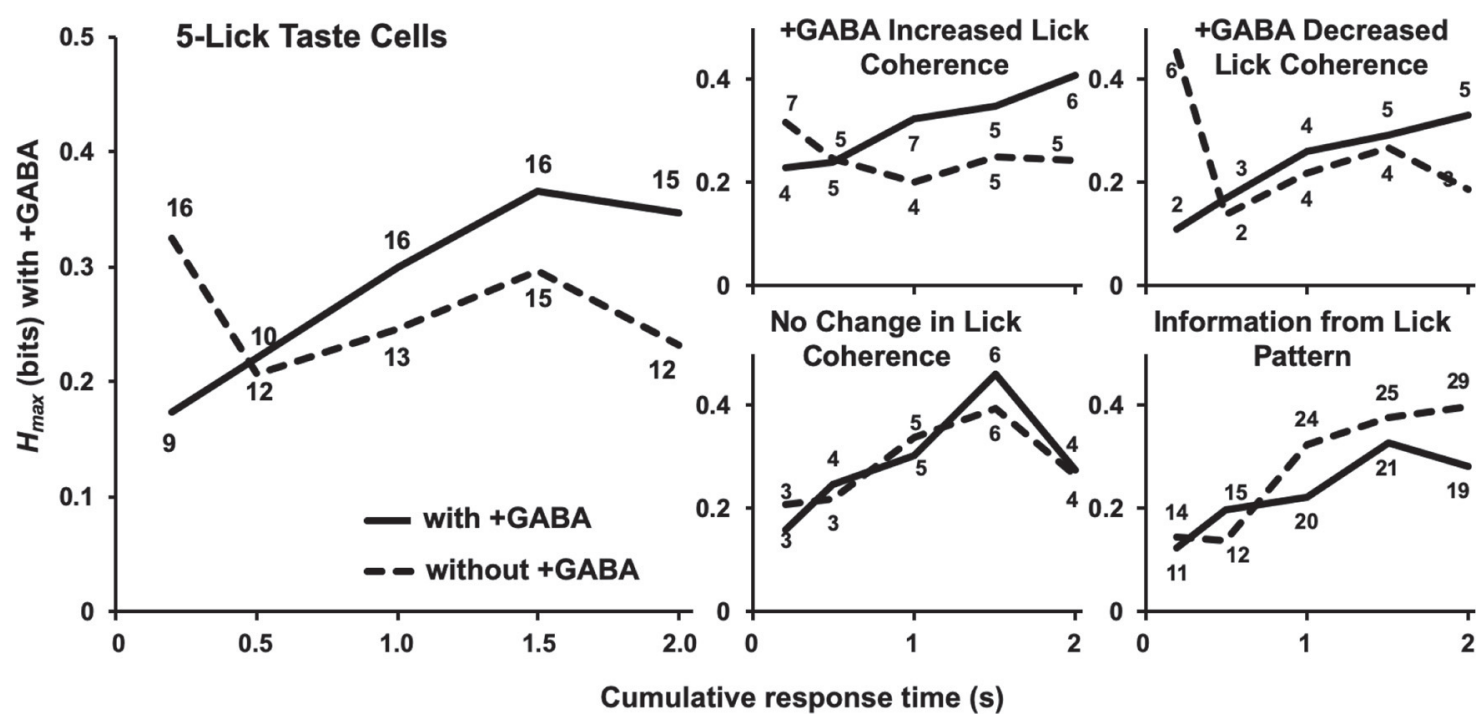

Figure 8. Information (in bits) conveyed about the five prototypical tastants from the population of rNTS neurons over the first $2 \mathrm{~s}$ of response. Separate analyses were conducted at each response interval. Temporal coding information obtained with (solid line) and without (dashed line) GABA stimulation is shown. Left, Information for all taste cells with five-lick responses. Right, Neurons were separated into groups depending on how GABA stimulation affected stimulus-restricted lick coherence. Also shown is the effect of GABA stimulation on the taste-related information conveyed by the lick pattern. Numbers adjacent to each data point denote the number of neurons in which significant taste quality information was obtained. GABA stimulation enhanced the information conveyed about salty tastes only in those cells where GABA also enhanced lick coherence. GABA stimulation did not affect the information conveyed by the lick pattern.

and performed MSA on the two groups. Once again, GABAergic stimulation increased information in the later time points (Fig. 10) with a maximum increase of 0.21 bits (118\%) at $1.5 \mathrm{~s}$. GABA-induced increase in palatability-related information was observed whether GABA activation increased lick coherence [maximum, 0.27 bits (246\%) at 1.5 s], decreased lick coherence [0.11 bits $(58 \%)$ at $1.5 \mathrm{~s}$ ] or had no effect on lick coherence [ 0.23 bits $(86 \%)$ at $1.5 \mathrm{~s}$ ], with a nonsignificant trend to greater increases in palatability-related information in those neurons in which GABA had a larger effect on lick coherence. Again, information conveyed solely by the lick pattern was not changed with GABAergic stimulation.

\section{Histology}

Lesion analysis shows that the electrodes were dispersed throughout the rNTS from 11.76 to 12.48 . Figure 11 shows the locations (asterisks) of the electrodes in each of the five rats from which data were collected. As is apparent, the lesions were mostly lateral with the most rostral lesion also being the most medial (Fig. 11A). Figure $11 B$ shows channelrhodopsin expression in the area surrounding the rNTS lesion. While there were many labeled neurons dorsal to the rNTS, the proximity of the fiber-optic implant to the recording electrode ensured that these extra-rNTS cells were not optogenetically activated (Yizhar et al., 2011). In addition, the labeled area dorsal to the rNTS is part of the vestibular nucleus, an area that is not known to send projections to the rNTS.

\section{Discussion}

Enhanced GABAergic tone in the rNTS remodeled the acrossneuron pattern of taste responsiveness and enhanced the information about taste quality and palatability conveyed by the temporal characteristics of the response in a subset of cells. In 


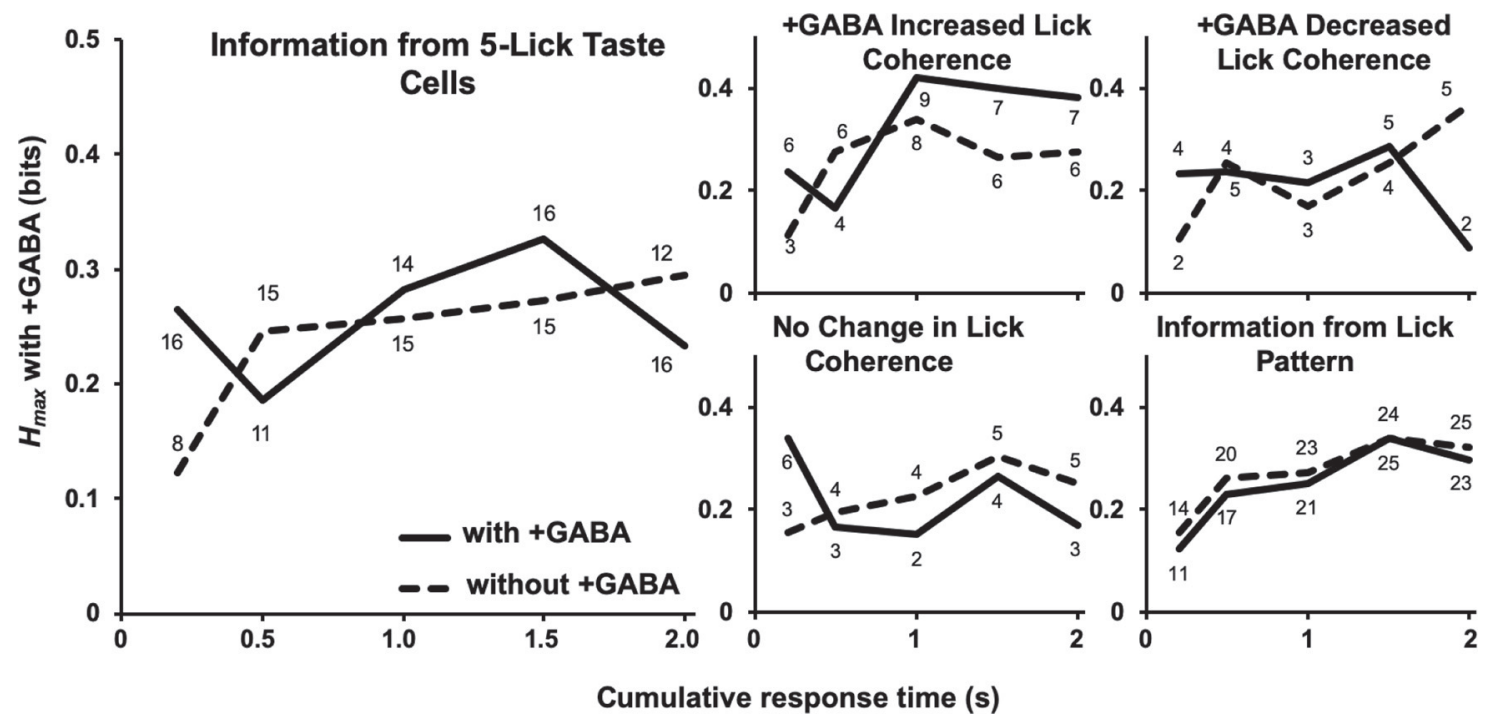

Figure 9. Information (in bits) conveyed about salty tastants $\left(\mathrm{NaCl}, \mathrm{KCl}\right.$, and $\left.\mathrm{NH}_{4} \mathrm{Cl}\right)$ from the population of $\mathrm{rNTS}$ neurons over the first $2 \mathrm{~s}$ of response. Separate analyses were conducted at each response interval. Temporal coding information obtained with (solid line) and without (dashed line) GABA stimulation is shown. Left, Information for all taste ells with five-lick responses. Right, Neurons were separated into groups depending on how GABA stimulation affected stimulus-restricted lick coherence. Also shown is the effect of GABA stimulation on the taste-related information conveyed by the lick pattern. Numbers adjacent to each data point denote number of neurons in which significant taste quality information was obtained. GABA stimulation enhanced the information conveyed about salty tastes only in those cells where GABA also enhanced lick coherence. GABA stimulation did not affect the information conveyed by the lick pattern.

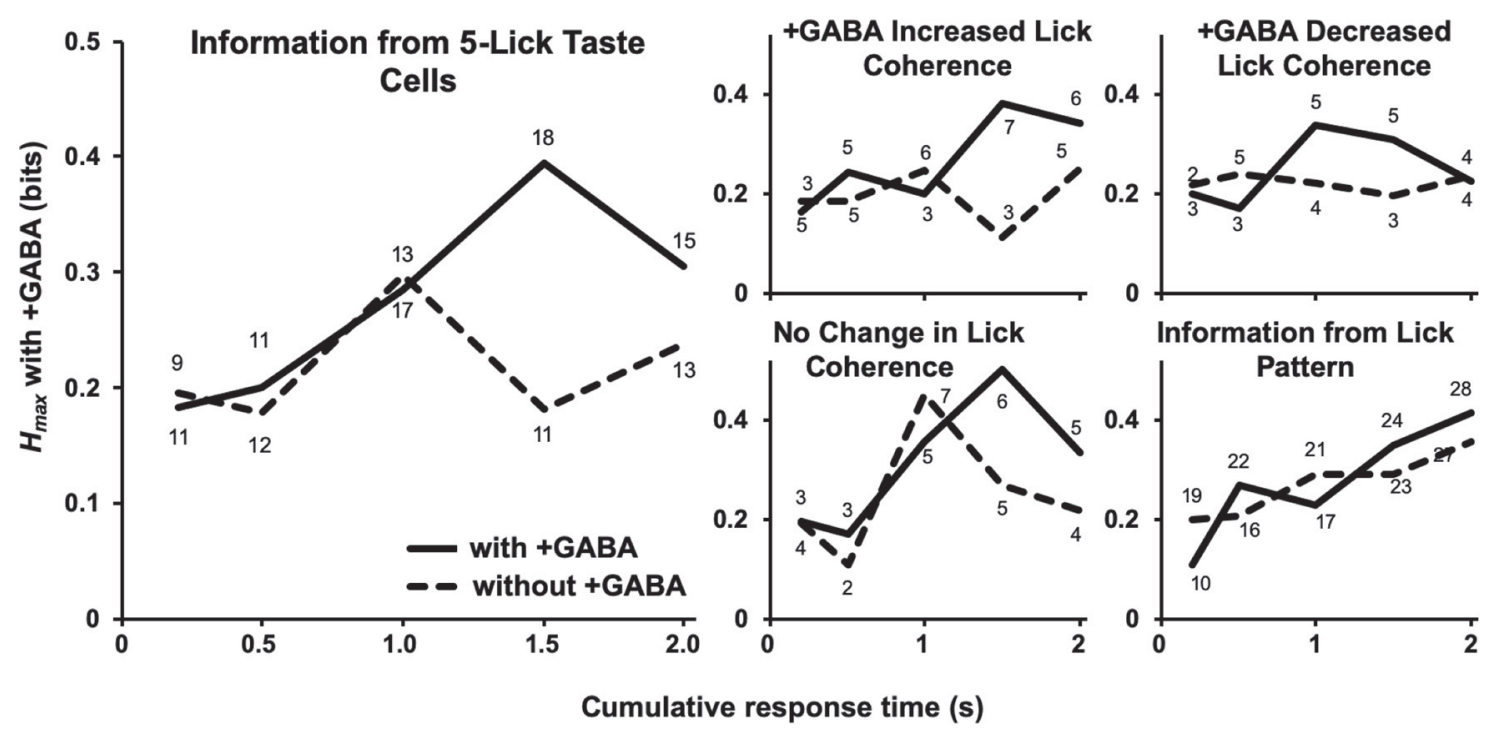

Figure 10. Information (in bits) conveyed about palatable (sucrose, NaCl) versus unpalatable (citric acid, quinine) from the population of rNTS neurons over the first $2 \mathrm{~s}$ of response. Separate analyses were conducted at each response interval. Temporal coding information obtained with (solid line) and without (dashed line) GABA stimulation is shown. Left, Information for all taste cells with five-lick responses. Right, Neurons were separated into groups depending on how GABA stimulation affected stimulus-restricted lick coherence. Also shown is the effect of GABA stimulation on the taste-related information conveyed by the lick pattern. Numbers adjacent to each data point denote number of neurons in which significant taste quality information was obtained. Regardless of the effect of GABA stimulation on lick coherence, GABA stimulation enhanced the information conveyed about taste palatability in taste responses $>1 \mathrm{~s}$. GABA stimulation did not affect the information conveyed by the lick pattern.

general, there was a great deal of diversity in the effects of GABA activation at the single-cell level. In individual cells in the rNTS taste response, profiles were changed by laser stimulation of GABA terminals in about half $(22$ of $54 ; 47 \%)$ of the sample of neurons. Responses to some stimuli were enhanced and others attenuated, sometimes within the same cell. In fact, there were neurons that responded to taste stimuli only during GABAergic stimulation $(n=2)$ and others that were rendered completely unresponsive to taste under GABAergic influence $(n=13)$. These GABA-induced cell-by-cell changes did not shift the overall interrelationship among response patterns but instead changed the identities of the cells that contributed to the across-unit patterns of response associated with each taste stimulus. Enhancing GABA did, however, change the temporal patterns of tasteevoked activity such that the information discriminating palatable (sucrose and $\mathrm{NaCl}$ ) versus unpalatable (citric acid and quinine) increased when longer taste responses (1-2 s) were considered.

The present results expand the results reported by Smith and Li (1998). In that study, either GABA or the GABA antagonist 


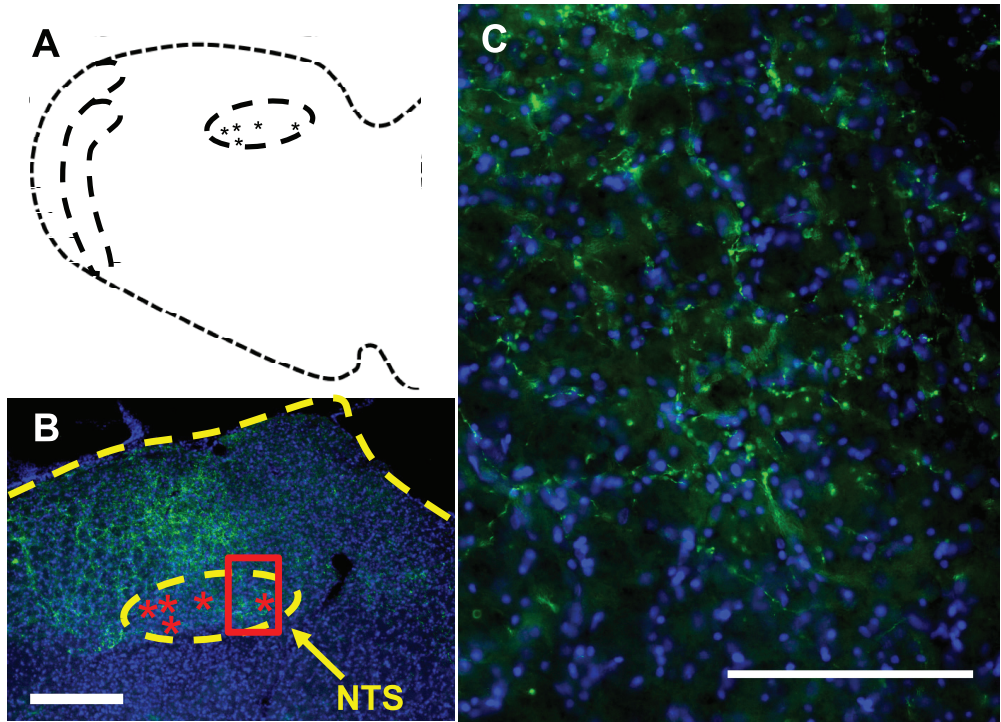

Figure 11. Histologic reconstruction of neuronal recordings and channelrhodopsin expression in the rNTS. $\boldsymbol{A}$, Schematic diagram of the brainstem with a dashed oval outlining the rNTS and the center of the lesion associated with each of the five rats from which data were collected are represented by an asterisk (*). Lesions ranged from 11.76 to $12.48 \mathrm{~mm}$ posterior to bregma. $\boldsymbol{B}$, Image of the rNTS (dashed yellow oval); red box represents the magnified inset. Scale bar, $500 \mu \mathrm{m}$. $\boldsymbol{C}$ Magnified image of rNTS. Channelrhodopsin, green; DAPI, blue. Scale bar, $100 \mu \mathrm{m}$.

bicuculline methiodide (BICM) was infused directly into the rNTS in urethane-anesthetized hamsters. Their data suggested that GABA narrows the tuning of taste-responsive cells. In awake rats in the present study, we confirmed that optogenetic activation of GABA in rNTS narrows taste tuning in a subset of cells; however, it also broadened the response profile in another subset of cells. While Smith and Li (1998) tested only the best and second best stimuli with GABA and BICM, we tested the effects of GABA enhancement for all of the basic tastants and found more complex effects. So, for example, it was not uncommon for GABA enhancement to attenuate the response to one stimulus and amplify the response to another in the same cell. In those cases, the breadth of tuning did not show a net change, though the complement of tastants that evoked a response was altered.

Results of the MDS analyses illustrate the effect of augmenting GABAergic tone on the population coding of taste in rNTS. Specifically, under the influence of enhanced GABA release, the configuration of the taste space generated by the across-unit pattern was essentially unchanged compared with the taste space without GABA enhancement. That is, in both taste spaces, patterns associated with the five basic taste qualities were well separated from each other. However, the placement of taste stimuli in the taste space with GABA enhancement was systematically shifted. This result implies that, for any given tastant, the identity of the cells that conveyed the signal was shifted by GABA enhancement, but the overall relationship between taste qualities, as signaled by the across-unit pattern, was essentially unchanged.

While GABA enhancement affected taste response magnitudes, it also modified the temporal arrangement of spikes within taste responses. Furthermore, these effects were correlated with GABA-induced changes in lick coherence. In general, GABA enhancement boosted the information conveyed about the five basic taste qualities. A closer analysis suggested that this effect was most prominent in those cells that showed an increase in GABA-induced lick coherence. Moreover, the information conveyed about $\mathrm{NaCl}, \mathrm{KCl}$, and $\mathrm{NH}_{4} \mathrm{Cl}$ was increased by GABA enhancement only in those cells where GABA also increased lick coherence. The largest effect of increasing GABAergic tone was seen in the discrimination of palatable (sucrose and $\mathrm{NaCl}$ ) versus unpalatable (citric acid and quinine) tastants. This effect was apparent regardless of the effect of GABA on lick coherencethough, again, it was more prominent in cells in which GABA enhanced lick coherence. Interestingly, this change was only manifested at longer taste response intervals (1-2 s), which is considered to be the critical period for judging palatability in the gustatory cortex (Katz et al., 2001); our finding of a similar time dependence in the brainstem suggests that the action of GABA in the brainstem may be involved in this effect.

Lick coherence is common in the brainstem taste areas of awake unrestrained animals (Roussin et al., 2012; Weiss et al., 2014; Denman et al., 2019); most NTS cells, including most taste-responsive cells, show some degree of lick coherence. We have found that, in addition to taste-responsive cells, non-taste-responsive cells that show significant lick coherence can also convey some information about taste quality (Roussin et al., 2012; Weiss et al., 2014; Denman et al., 2019). Thus, the lick pattern, as reflected in the lick-coherent spiking of these cells, can buttress the information about taste quality conveyed by taste-evoked activity. GABA enhancement in NTS was found to alter lick coherence during taste stimulus presentation only in taste-responsive cells. Thus, our data suggest that GABAergic activity may modulate taste-related lick coherence to amplify the contributions of some cells while diminishing the contributions of others to the neural representation of taste in the rNTS. Collectively, these effects essentially reconfigure the sensorimotor balance among taste-responsive neurons in rNTS.

The effects of GABA activation reported here must be considered in the context of some obvious limitations. For example, the amplification of GABAergic tone via optogenetic stimulation is a nonphysiological manipulation. Under normal physiological conditions, cells in the rNTS are under a tonic inhibitory influence, with GABA as a major contributor (Liu et al., 1993; Smith and Li, 1998; Grabauskas and Bradley, 2003). Moreover, taste simulation may evoke GABA release in rNTS. Experimental augmentation of GABA release during taste stimulation represents at best a crude exaggeration of the natural influence of GABA on rNTS cells. Nevertheless, the fact that there were consistent effects on taste responsivity within individual cells and systematic effects on a population level implies that there are meaningful concepts that can be derived from our results.

The fact that global optogenetic activation of GABA activates GABA release from a variety of sources represents another limitation of the present study and could be in part responsible for the diversity of effects seen. GABAergic projections arise from both local interneurons in NTS (Lasiter and Kachele, 1988; Davis, 1993) as well as centrifugal structures such as the GC (Torrealba and Müller, 1996; Smith and Li, 2000) or AMG (Batten et al., 2002; Saha et al., 2002). Further, stimulation of the solitary tract can monosynaptically activate GABAergic NTS cells (Boxwell et al., 2013), suggesting that afferent input can initiate feedforward inhibition. Although input from the gustatory cortex is mainly glutaminergic (Torrealba and Müller, 1996), some 
cortical input to the NTS makes connections to GABAergic interneurons (Smith and Li, 2000). Temporary pharmacological elimination of GC input to NTS shows similar effects to that reported here: responses to some tastants were attenuated, while others were enhanced, sometimes within the same cell (Di Lorenzo and Monroe, 1995). These data suggest that the effects of GABA activation may be at least partially accounted for by mimicking GC input to NTS. Another potential source of GABAergic influence may be the AMG. While anatomic evidence suggests that AMG input to the NTS is inhibitory (Batten et al., 2002; Saha et al., 2002), physiological studies suggest that the effect of stimulation of AMG-NTS input is excitatory (Cho et al., 2003), suggesting the possibility that the AMG generates a disinhibitory effect in the NTS (Herman et al., 2012). If true, that might contribute to the enhanced responses that became apparent following enhanced GABA release. Since the AMG supplies a rich centrifugal innervation to the rostral NTS (Kang and Lundy, 2009), GABA-induced enhancement of information about palatable versus unpalatable tastants might mainly be because of GABA release from AMG-NTS projections.

The effects of GABA release during taste stimulation was studied in the NTS of awake, unrestrained rats. GABA changed the taste response profile in about half of the taste-responsive cells that were recorded, but the overall interrelationships among the taste-evoked across-unit patterns were not altered. Interestingly, GABA activation did not result in more narrow tuning in taste-responsive cells as might have been predicted from studies conducted in anesthetized animals (Smith and Li, 1998). Instead, the population response was essentially remodeled by shifting the identities of the cells conveying specific stimulus-related signals. The coherence of spike activity with the lick pattern was also altered by GABA activation but primarily in taste-responsive cells. In those cells where GABA activation enhanced taste-related lick coherence, information conveyed by temporal coding about taste quality was increased. Most notably, taste-driven GABA activation increased the information conveyed by the temporal characteristics of taste responses about palatability, especially in neurons with GABA-induced shifts in lick coherence. In all, this study shows that GABAergic activation remodels the global population response to taste by both shifts in the responses to taste and the extent to which neural activity reflects licking. Future experiments should tease apart the effects of the various sources of GABAergic activity to obtain a more precise picture of the role of GABA in the rostral NTS.

\section{References}

Batten TF, Gamboa-Esteves FO, Saha S (2002) Evidence for peptide co-transmission in retrograde- and anterograde-labelled central nucleus of amygdala neurones projecting to NTS. Auton Neurosci 98:28-32.

Benjamini Y, Hochberg Y (1995) Controlling the false discovery rate: a practical and powerful approach to multiple testing. J R Stat Soc Series B Stat Methodol 57:289-300.

Boxwell AJ, Yanagawa Y, Travers SP, Travers JB (2013) The $\mu$-opioid receptor agonist DAMGO presynaptically suppresses solitary tract-evoked input to neurons in the rostral solitary nucleus. J Neurophysiol 109:28152826.

Breza JM, Nikonov AA, Contreras RJ (2010) Response latency to lingual taste stimulation distinguishes neuron types within the geniculate ganglion. J Neurophysiol 103:1771-1784.

Cho YK, Li CS, Smith DV (2002) Taste responses of neurons of the hamster solitary nucleus are enhanced by lateral hypothalamic stimulation. J Neurophysiol 87:1981-1992.

Cho YK, Li CS, Smith DV (2003) Descending influences from the lateral hypothalamus and amygdala converge onto medullary taste neurons. Chem Senses 28:155-171.
Davis BJ (1993) GABA-like immunoreactivity in the gustatory zone of the nucleus of the solitary tract in the hamster: light and electron microscopic studies. Brain Res Bull 30:69-77.

Denman AJ, Sammons JD, Victor JD, Di Lorenzo PM (2019) Heterogeneity of neuronal responses in the nucleus of the solitary tract suggests sensorimotor integration in the neural code for taste. J Neurophysiol 121:634645.

Di Lorenzo PM, Lemon CH (2000) The neural code for taste in the nucleus of the solitary tract of the rat: effects of adaptation. Brain Res 852:383397.

Di Lorenzo PM, Monroe S (1995) Corticofugal influence on taste responses in the nucleus of the solitary tract in the rat. J Neurophysiol 74:258-272.

Di Lorenzo PM, Lemon CH, Reich CG (2003) Dynamic coding of taste stimuli in the brain stem: effects of brief pulses of taste stimuli on subsequent taste responses. J Neurosci 23:8893-8902.

Escanilla OD, Victor JD, Di Lorenzo PM (2015) Odor-taste convergence in the nucleus of the solitary tract of the awake freely licking rat. J Neurosci 35:6284-6297.

Gompf HF, Budygin EA, Fuller PM, Bass CE (2015) Targeted genetic manipulations of neuronal subtypes using promoter-specific combinatorial AAVs in wild-type animals. Front Behav Neurosci 9:152.

Grabauskas G, Bradley RM (1998) Tetanic stimulation induces short-term potentiation of inhibitory synaptic activity in the rostral nucleus of the solitary tract. J Neurophysiol 79:595-604.

Grabauskas G, Bradley RM (1999) Potentiation of GABAergic synaptic transmission in the rostral nucleus of the solitary tract. Neuroscience 94:11731182.

Grabauskas G, Bradley RM (2003) Frequency-dependent properties of inhibitory synapses in the rostral nucleus of the solitary tract. J Neurophysiol 89:199-211.

Herman MA, Gillis RA, Vicini S, Dretchen KL, Sahibzada N (2012) Tonic GABAA receptor conductance in medial subnucleus of the tractus solitarius neurons is inhibited by activation of mu-opioid receptors. J Neurophysiol 107:1022-1031.

Hirata S-i, Nakamura T, Ifuku H, Ogawa H (2005) Gustatory coding in the precentral extension of area 3 in Japanese macaque monkeys; comparison with area G. Exp Brain Res 165:435-446.

Kang Y, Lundy RF (2009) Terminal field specificity of forebrain efferent axons to brainstem gustatory nuclei. Brain Res 1248:76-85.

Kattla S, Lowery MM (2010) Fatigue related changes in electromyographic coherence between synergistic hand muscles. Exp Brain Res 202:89-99.

Katz DB, Simon SA, Nicolelis MA (2001) Dynamic and multimodal responses of gustatory cortical neurons in awake rats. J Neurosci 21:4478-4489.

Lasiter PS, Kachele DL (1988) Organization of GABA and GABA-transaminase containing neurons in the gustatory zone of the nucleus of the solitary tract. Brain Res Bull 21:623-636.

Leonard NL, Renehan WE, Schweitzer L (1999) Structure and function of gustatory neurons in the nucleus of the solitary tract. IV. The morphology and synaptology of GABA-immunoreactive terminals. Neurosci 92:151-162.

Li CS, Cho YK, Smith DV (2002) Taste responses of neurons in the hamster solitary nucleus are modulated by the central nucleus of the amygdala. J Neurophysiol 88:2979-2992.

Liu H, Behbehani M, Smith DV (1993) The influence of GABA on cells in the gustatory region of the solitary nucleus. Chem Senses 18:285- 305.

Matsuo R, Shimizu N, Kusano K (1984) Lateral hypothalamic modulation of oral sensory afferent activity in nucleus tractus solitarius neurons of rats. J Neurosci 4:1201-1207.

Monroe S, Di Lorenzo PM (1995) Taste responses in neurons in the nucleus of the solitary tract that do and do not project to the parabrachial pons. J Neurophysiol 74:249-257.

Mukherjee N, Wachutka J, Katz DB (2017) Python meets systems neuroscience: affordable, scalable and open-source electrophysiology in awake, behaving rodents. Paper presented at the 16th Python in Science Conference (SciPy 2017), Austin, TX, July.

Murzi E, Hernandez L, Baptista T (1986) Lateral hypothalamic sites eliciting eating affect medullary taste neurons in rats. Physiol Behav 36:829-834.

Panzeri S, Senatore R, Montemurro MA, Petersen RS (2007) Correcting for the sampling bias problem in spike train information measures. J Neurophysiol 98:1064-1072. 
Rainer G, Asaad WF, Miller EK (1998) Selective representation of relevant information by neurons in the primate prefrontal cortex. Nature 393:577-579.

Rosen AM, Di Lorenzo PM (2009) Two types of inhibitory influences target different groups of taste-responsive cells in the nucleus of the solitary tract of the rat. Brain Res 1275:24-32.

Roussin AT, D'Agostino AE, Fooden AM, Victor JD, Di Lorenzo PM (2012) Taste coding in the nucleus of the solitary tract of the awake, freely licking rat. J Neurosci 32:10494-10506.

Saha S, Henderson Z, Batten TF (2002) Somatostatin immunoreactivity in axon terminals in rat nucleus tractus solitarii arising from central nucleus of amygdala: coexistence with GABA and postsynaptic expression of sst2A receptor. J Chem Neuroanat 24:1-13.

Sammons JD, Weiss MS, Escanilla OD, Fooden AF, Victor JD, Di Lorenzo PM (2016) Spontaneous changes in taste sensitivity of single units recorded over consecutive days in the brainstem of the awake rat. PLoS One 11:e0160143.

Smith DV, Li C-S (1998) Tonic GABAergic inhibition of taste-responsive neurons in the nucleus of the solitary tract. Chem Senses 23:159-169.

Smith DV, Li C-S (2000) GABA-mediated corticofugal inhibition of tasteresponsive neurons in the nucleus of the solitary tract. Brain Res 858:408-415.

Smith DV, Travers JB (1979) A metric for the breadth of tuning of gustatory neurons. Chem Senses 4:215-229.
Smith DV, Li C-S, Davis BJ (1998) Excitatory and inhibitory modulation of taste responses in the hamster brainstem. Ann N Y Acad Sci 855:450456.

Torrealba F, Müller C (1996) Glutamate immunoreactivity of insular cortex afferents to the nucleus tractus solitarius in the rat: a quantitative electron microscopic study. Neurosci 71:77-87.

Victor JD, Purpura KP (1996) Nature and precision of temporal coding in visual cortex: a metric-space analysis. J Neurophysiol 76:1310-1326.

Victor JD, Purpura KP (1997) Sensory coding in cortical neurons. Recent results and speculations. Ann N Y Acad Sci 835:330-352.

Wakabayashi KT, Feja M, Baindur AN, Bruno MJ, Bhimani RV, Park J, Hausknecht K, Shen R-Y, Haj-Dahmane S, Bass CE (2019) Chemogenetic activation of ventral tegmental area GABA neurons, but not mesoaccumbal GABA terminals, disrupts responding to reward-predictive cues. Neuropsychopharmacology 44:372-380.

Weiss MS, Victor JD, Di Lorenzo PM (2014) Taste coding in the parabrachial nucleus of the pons in awake, freely licking rats and comparison with the nucleus of the solitary tract. J Neurophysiol 111:1655-1670.

Xiao X, Li J, Samulski RJ (1998) Production of high-titer recombinant adeno-associated virus vectors in the absence of helper adenovirus. J Virol 72:2224-2232.

Yizhar O, Fenno LE, Davidson TJ, Mogri M, Deisseroth K (2011) Optogenetics in neural systems. Neuron 71:9-34 